\title{
Fundamental Limits of Decentralized Caching in Fog-RANs with Wireless Fronthaul
}

\author{
Fan $\mathrm{Xu}$ and Meixia Tao \\ Department of Electronic Engineering, Shanghai Jiao Tong University, Shanghai, China \\ Emails: \{xxiaof, mxtao\}@sjtu.edu.cn
}

\begin{abstract}
This paper aims to characterize the synergy of distributed caching and wireless fronthaul in a fog radio access network (Fog-RAN) where all edge nodes (ENs) and user equipments (UEs) have a local cache and store contents independently at random. The network operates in two phases, a file-splitting based decentralized cache placement phase and a fronthaul-aided content delivery phase. We adopt normalized delivery time (NDT) to characterize the asymptotic latency performance with respect to cache size and fronthaul capacity. Both an achievable upper bound and a theoretical lower bound of NDT are obtained, and their multiplicative gap is within 12. In the proposed delivery scheme, we utilize the fronthaul link, by exploiting coded multicasting, to fetch both non-cached and cached contents to boost EN cooperation in the access link. In particular, to fetch contents already cached at ENs, an additional layer of coded multicasting is added on the coded messages desired by UEs in the fronthaul link. Our analysis shows that the proposed delivery scheme can balance the delivery latency between the fronthaul link and access link, and is approximately optimum under decentralized caching.
\end{abstract}

\section{INTRODUCTION}

Caching is emerging as an effective technique to reduce peak-hour data traffic and improve user perceived experience in wireless networks. Unlike traditional web-caching and innetwork caching, caching at the edge of wireless networks is able to exploit the broadcast nature of wireless medium and thus achieve global caching gain [1]. Recently, it has attracted many interests to characterize the fundamental limits of caching in various wireless networks. This work aims to advance this topic by studying the synergy between distributed caching and wireless fronthauling in fog radio access networks (Fog-RANs).

Previously, the gain of caching is studied in wireless interference networks where caches are equipped at all transmitters and receivers [2]-[5]. It is found in [2] that with a generic file splitting and caching strategy, the interference network topology can be changed into a new family of channels, referred to as cooperative $X$-multicast channels, and hence leverage transmitter cooperation gain and coded multicasting gain, apart from receiver local caching gain. These works [2][5], however, have assumed that there exists a central controller that coordinates the file splitting and cache placement among

This work is supported by the National Natural Science Foundation of China under grants 61571299 and 61521062. all distributed nodes (at least all the transmit nodes if not receive nodes as in [5]). Moreover, they also assume that the total cache size among the network nodes is large enough to collectively store the entire database without cache miss.

To exploit the potential of practical and scalable caching in large and random networks, decentralized coded caching is proposed at the user side where users can independently cache file bits at random [6]. It is shown in [6] that decentralized coded caching can achieve a performance close to the optimal centralized scheme.

The idea of decentralized coded caching can be extended to a general interference network where all the transmitters and receivers cache file bits independently at random. However, due to the lack of a central controller for careful cache placement, it is very likely to have cache miss even when the total cache size is as large as in the centralized scheme. To overcome the cache miss issue, the works [7], [8] consider a Fog-RAN where each cache-enabled edge node $(\mathrm{EN})$ is connected via a fronthaul link to a cloud server which has access to the entire database. They characterize a latencyoriented performance with respect to both the EN cache size and the fronthaul capacity. The works [9]-[11] consider a Fog-RAN where all ENs and user equipments (UEs) are equipped with local caches. The authors in [9] propose a caching-and-delivery scheme that combines network-coded fronthaul transmission and cache-aided interference management. The authors in [10] propose a mixed cache placement, i.e., centralized caching at ENs and decentralized caching at UEs, and employ a combination of interference management techniques in the delivery phase. The authors in [11] consider a decentralized cache placement at all ENs and UEs and propose a coded delivery strategy that exploits the network topology for Fog-RANs. Note that [11] is only limited to two ENs only.

The contribution of this work is to characterize the latency performance of a Fog-RAN with wireless fronthaul and for arbitrary number of ENs and UEs, where all ENs and UEs are equipped with caches. Considering the random mobility of UEs and the dynamic on/off of ENs, we apply decentralized cache placement at all ENs and UEs without central coordination. As in [2], [5], [7]-[11], we adopt normalized delivery time (NDT) as the performance metric. The network operates in two phases, a decentralized cache placement phase 
and a fronthaul-aided content delivery phase. In our proposed delivery scheme, the wireless fronthaul is not only responsible to fetch cache-miss contents but also can be used to fetch contents already cached at ENs to boost transmission cooperation to any desired level in the access link. To fetch contents already cached at ENs, an additional layer of coded multicasting on top of the coded messages desired by UEs is exploited in the fronthaul link. To fetch contents not cached at ENs, the coded messages, rather than the original files desired by UEs, are transmitted in the fronthaul link. The access transmission in our proposed delivery scheme is similar to [2], which transforms the access link into the cooperative $\mathrm{X}$-multicast channel. Based on the proposed delivery scheme, we obtain an achievable upper bound of the minimum NDT of the network with decentralized caching. Numerical results show that our NDT performance is even better than that using centralized caching [9] with wireless fronthaul and that using centralized caching [7], mixed caching [10], and decentralized caching [11] with dedicated fronthaul under certain conditions. Under decentralized caching, we also obtain a theoretical lower bound of the minimum NDT by applying cut-set-like bounds in the fronthaul transmission and access transmission separately. It is shown that the multiplicative gap between the upper and lower bounds is within 12.

Notations: $[K]$ denotes the set $\{1,2, \ldots, K\} . \mathcal{C N}(0,1)$ denotes the complex-valued Gaussian distribution with zero mean and unit variance.

\section{System Model And PRoblem Description}

\section{A. Fog-RAN with Wireless Fronthaul}

We consider a Fog-RAN as shown in Fig 1, where there are $N_{T}\left(N_{T} \geq 2\right)$ ENs, $N_{R}\left(N_{R} \geq 2\right)$ UEs, and the ENs are connected to a macro base station (MBS), or a cloud server, through a shared wireless fronthaul link. All ENs and UEs have a local cache each. The access link between each EN and each UE experiences channel fading, and is corrupted with additive white Gaussian noise. The communication at each time slot $t$ over the access channel is modeled by

$$
Y_{q}(t)=\sum_{p=1}^{N_{T}} h_{q p}(t) X_{p}(t)+Z_{q}(t), q \in\left[N_{R}\right],
$$

where $Y_{q}(t) \in \mathbb{C}$ is the received signal at UE $q, X_{p}(t) \in \mathbb{C}$ is the transmitted signal at $\mathrm{EN} p, h_{q p}(t) \in \mathbb{C}$ is the channel coefficient from $\mathrm{EN} p$ to UE $q$ which is assumed to be independent and identically distributed (i.i.d.) as some continuous distribution, and $Z_{q}(t)$ is the noise at UE $q$ distributed as $\mathcal{C N}(0,1)$.

The fronthaul link between the MBS and all the ENs also experiences channel fading and additive white Gaussian noise. The communication at each time slot $t$ over the fronthaul channel is modeled by

$$
Q_{p}(t)=g_{p}(t) S(t)+N_{p}(t), p \in\left[N_{T}\right],
$$

where $Q_{p}(t) \in \mathbb{C}$ is the received signal at $\mathrm{EN} p, S(t) \in \mathbb{C}$ is the transmitted signal from the MBS, $g_{p}(t) \in \mathbb{C}$ is the channel

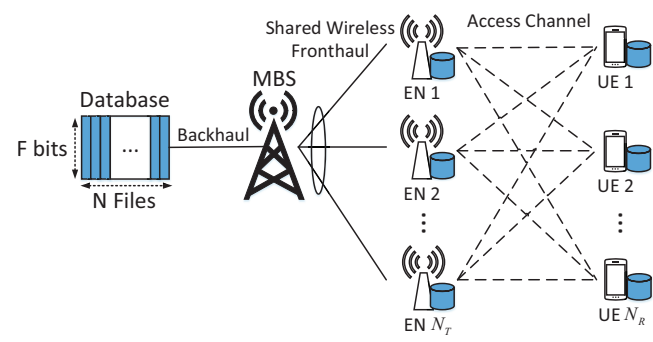

Fig. 1: Cache-aided Fog-RAN.

coefficient from the MBS to EN $p$ which is assumed to be i.i.d. as some continuous distribution, and $N_{p}(t)$ is the noise at EN $p$ distributed as $\mathcal{C N}(0,1)$.

Consider a database consisting of $N$ files, denoted as $\left\{W_{1}, W_{2}, \ldots, W_{N}\right\}$, each with $F$ bits. Throughout this study, we consider $N \geq N_{R}$ so that each UE can request a distinct file. The MBS has full access to the database via a dedicated backhaul link. Each EN can store $\mu_{T} N F\left(\mu_{T} \leq 1\right)$ bits locally, and each UE can store $\mu_{R} N F\left(\mu_{R} \leq 1\right)$ bits locally, where $\mu_{T}$ and $\mu_{R}$ are referred to as normalized cache sizes at each EN and UE, respectively. In this work, we consider the complete region for the normalized cache sizes, i.e., $0 \leq \mu_{R}, \mu_{T} \leq 1$, because of the presence of the fronthaul. Note that the works [2], [5] only consider the feasible region $N_{T} \mu_{T}+\mu_{R} \geq 1$, while [3], [4] only consider $N_{T} \mu_{T} \geq 1$.

The network operates in two phases, a decentralized cache placement phase and a two-hop content delivery phase, as detailed in the next two subsections.

\section{B. Decentralized Cache Placement}

We adopt decentralized cache placement at all ENs and UEs. Each EN $p$ (or UE $q$ ) independently caches a subset of $\mu_{T} F$ (or $\mu_{R} F$ ) bits of each file $W_{n}$, chosen uniformly at random, denoted as $U_{p}^{n}$ (or $V_{q}^{n}$ ), for $n \in[N]$. Denote $U_{p} \triangleq \bigcup_{n \in[N]} U_{p}^{n}$ (or $V_{q} \triangleq \bigcup_{n \in[N]} V_{q}^{n}$ ) as all the cached bits at EN $p$ (or UE $q$ ). Note that neither inter-file nor intra-file coding is allowed in the considered decentralized cache placement phase.

By the law of large numbers, when file size $F$ is large enough, the size of each subfile cached exactly at an arbitrary set of $m$ UEs $\left(0 \leq m \leq N_{R}\right)$ and an arbitrary set of $n$ ENs $(0 \leq$ $\left.n \leq N_{T}\right)$ is $\mu_{R}^{m}\left(1-\mu_{R}\right)^{N_{R}-m} \mu_{T}^{n}\left(1-\mu_{T}\right)^{N_{T}-n} F+o(F)$ bits with high probability. Since this paper focuses on the extreme case when $F \rightarrow \infty$, we ignore the $o(F)$ term in the rest of the paper, and define

$$
f_{m, n} \triangleq \mu_{R}^{m}\left(1-\mu_{R}\right)^{N_{R}-m} \mu_{T}^{n}\left(1-\mu_{T}\right)^{N_{T}-n}
$$

as the fractional size of each subfile cached exactly at each node set with $m$ UEs and $n$ ENs, similar to [6].

\section{Two-Hop Content Delivery}

Each UE $q$ requests a file $W_{d_{q}}$ from the database. We denote $\mathbf{d} \triangleq\left(d_{q}\right)_{q=1}^{N_{R}} \in[N]^{N_{R}}$ as the demand vector from all the $N_{R}$ UEs. The content delivery phase is a two-hop transmission process, the first hop being the fronthaul channel and the second hop being the access channel. 
1) Fronthaul Transmission: The MBS employs an encoding function $\Lambda_{C}$ to map the entire database, UE demand $\mathbf{d}$, and channel realization $\mathbf{G}_{F} \triangleq\left\{g_{p}(t): \forall p \in\left[N_{T}\right], \forall t \in\left[T_{F}\right]\right\}$ to a length- $T_{F}$ codeword $(S[t]]_{t=1}^{T_{F}}$ with an average transmit power constraint $P^{r}$, i.e., $\frac{1}{T_{F}} \sum_{t=1}^{T_{F}=1}|S(t)|^{2} \leq P^{r}$, where $r>0$ is the power scaling of the fronthaul link compared to the access link with power $P$. Note that $r$ can also be viewed as the multiplexing gain in the fronthaul link [11].

2) Access Transmission: In this paper, we assume that all the ENs are half-duplex, which means that they cannot transmit over the access link while receiving from the fronthaul link at the same time. Thus, the fronthaul transmission and the access transmission take place in serial. After receiving signals from the fronthaul link, each EN $p$ uses an encoding function $\Lambda_{p}$ to map its cached content $U_{p}$, UE demand $\mathbf{d}$, received signals $\left(Q_{p}[t]\right)_{t=1}^{T_{F}}$, and channel realizations $\mathbf{G}_{F}$ and $\mathbf{H}_{A} \triangleq\left\{h_{q p}(t): \forall q \in\left[N_{R}\right], \forall p \in\left[N_{T}\right], \forall t \in\left[T_{A}\right]\right\}$ to a length- $T_{A}$ codeword $\left(X_{p}[t]\right)_{t=1}^{T_{A}}$. Note that $T_{F}$ and $T_{A}$ may depend on the UE demand $\mathbf{d}$ and channel realizations $\mathbf{G}_{F}$ and $\mathbf{H}_{A}$. Each codeword $\left(X_{p}[t]\right)_{t=1}^{T_{A}}$ has an average transmit power constraint $P$, i.e., $\frac{1}{T_{A}} \sum_{t=1}^{T_{A}}\left|X_{p}(t)\right|^{2} \leq P$.

Upon receiving signals $\left(Y_{q}[t]\right)_{t=1}^{T_{A}}$ in the access link, each UE $q$ employs a decoding function $\Gamma_{q}$ to decode $\hat{W}_{d_{q}}$ of its desired file $W_{d_{q}}$ from $\left(Y_{q}[t]\right)_{t=1}^{T_{A}}$ along with its cached content $V_{q}$, UE demand $\mathbf{d}$, and channel realizations $\mathbf{G}_{F}$ and $\mathbf{H}_{A}$ as side information.

Define $P_{\epsilon} \triangleq \max _{\mathbf{d}} \max _{q} \mathbb{P}\left(\hat{W}_{d_{q}} \neq W_{d_{q}}\right)$ as the worst-case error probability. A given set of coding functions $\left\{\Lambda_{C}, \Lambda_{p}, \Gamma_{q}\right.$ : $\left.p \in\left[N_{T}\right], q \in\left[N_{R}\right]\right\}$ in the delivery phase is said to be feasible if, for almost all channel realizations, $P_{\epsilon} \rightarrow 0$ when $F \rightarrow \infty$.

\section{Performance Metric}

Following [7], we adopt normalized delivery time (NDT) as the performance metric which is given by 1

$$
\tau\left(\mu_{R}, \mu_{T}, r\right) \triangleq \lim _{P \rightarrow \infty} \lim _{F \rightarrow \infty} \sup \frac{\max _{\mathbf{d}}\left(T_{F}+T_{A}\right)}{F / \log P} .
$$

We are interested in characterizing the minimum NDT of the network with decentralized caching which is defined as $\tau^{*}\left(\mu_{R}, \mu_{T}, r\right)=\inf \left\{\tau\left(\mu_{R}, \mu_{T}, r\right): \tau\left(\mu_{R}, \mu_{T}, r\right)\right.$ is achievable $\}$.

Remark 1. Similar to [9], given the two-hop content delivery phase, the NDT can be rewritten as $\tau=\tau_{F}+\tau_{A}$, where $\tau_{F}$ and $\tau_{A}$ is the NDT in the fronthaul link and access link, respectively. Based on the power constraint of fronthaul codeword $(S[t])_{t=1}^{T_{F}}$, the fronthaul link carries $r \log P$ bits per channel use in the high SNR regime. Denote $R_{F}$ as the sum traffic load normalized by file size $F$ in the fronthaul link. We can rewrite $\tau_{F}$ as $\tau_{F}=R_{F} / r$. Similar to [2, Remark 1], denote $R_{A}$ as the per-user traffic load normalized by file size $F$ and $d$ as the per-user degrees of freedom (DoF) in the access link. We can rewrite $\tau_{A}$ as $\tau_{A}=R_{A} / d$. Therefore, the NDT can be expressed more conveniently as

$$
\tau=\tau_{F}+\tau_{A}=R_{F} / r+R_{A} / d .
$$

\footnotetext{
${ }^{1}$ The same metric is also defined in [12] but under a different name.
}

\section{DELIVERY SCHEME FOR $3 \times 3$ FOG-RAN}

In this section, we use a Fog-RAN with $N_{T}=N_{R}=3$ to illustrate the proposed delivery scheme. The scheme can be easily generated to a general Fog-RAN with arbitrary $N_{T}$ and arbitrary $N_{R}$, which is given in Section IV] We consider the worst-case scenario that each UE requests a distinct file. Note that when some UEs request the same file, the proposed delivery scheme can still be applied by treating the requests as being different. Without loss of generality, we assume that UE $q$ desires $W_{q}$, for $q \in[3]$. We denote $W_{q, \Phi, \Psi}$ as the subfile desired by UE $q$ and cached at UE set $\Phi$ and EN set $\Psi$. Its fractional size is given by $f_{|\Phi|,|\Psi|}=\mu_{R}^{|\Phi|}\left(1-\mu_{R}\right)^{3-|\Phi|} \mu_{T}^{|\Psi|}(1-$ $\left.\mu_{T}\right)^{3-|\Psi|}$ based on (1).

Excluding the locally cached subfiles, each UE $q$, for $q \in[3]$, wants subfiles $\left\{W_{q, \Phi, \Psi}: \Phi \not \supset q, \Phi \subseteq[3], \Psi \subseteq[3]\right\}$. We divide the subfiles wanted by all UEs into different groups according to the size of $\Phi$ and $\Psi$, indexed by $\{(m, n): m \in$ $[2] \cup\{0\}, n \in[3] \cup\{0\}\}$, such that subfiles in group $(m, n)$ are cached at $m$ UEs and $n$ ENs. There are $3\left(\begin{array}{c}2 \\ m\end{array}\right)\left(\begin{array}{l}3 \\ n\end{array}\right)$ subfiles in group $(m, n)$. Each group of subfiles is delivered individually in the time division manner. In the following, we present the delivery strategy of two representative groups, $(m, 0)$ and $(m, 1)$, where $m \in[2] \cup\{0\}$. Before that, let us introduce the cooperative X-multicast channel defined in [2, Definition 2] which shall be mentioned throughout this section and Section IV

Definition 1 ( [2]). The channel characterized as follows is referred to as the $\left(\begin{array}{c}N_{T} \\ j\end{array}\right) \times\left(\begin{array}{c}N_{R} \\ m+1\end{array}\right)$ cooperative $\mathrm{X}$-multicast channel:

1) there are $N_{R}$ UEs and $N_{T}$ ENs;

2) each set of $m+1\left(m<N_{R}\right)$ UEs forms a UE multicast group;

3) each set of $j\left(j \leq N_{T}\right)$ ENs forms a EN cooperation group;

4) each EN cooperation group has an independent message for each UE multicast group.

\section{A. Delivery of Group $(m, 0)$}

Each subfile in group $(m, 0)$ is desired by one UE, cached at $m$ other UEs but none of ENs. Coded multicasting can be exploited through bit-wise XOR, similar to [1]. In specific, the set of coded messages is given by

$$
\left\{W_{\Phi^{+}, \emptyset}^{\oplus} \triangleq \bigoplus_{q \in \Phi^{+}} W_{q, \Phi^{+} \backslash\{q\}, \emptyset}: \Phi^{+} \subseteq[3],\left|\Phi^{+}\right|=m+1\right\} .
$$

In this work, we focus on the case with $F \rightarrow \infty$ for analytical tractability. By the law of large numbers, each coded message $W_{\Phi^{+}, \emptyset}^{\oplus}$ has $f_{m, 0} F$ bits, and is desired by UE set $\Phi^{+}$. Since the ENs do not have the coded messages in (4), these messages need to be generated at the MBS and then delivered to UEs via two compulsory hops, the fronthaul link and the access link. In the fronthaul link, we let the MBS naively multicast 
each coded message in (4) one by one to all three ENs. Thus, from Remark 1 the NDT of the fronthaul link is given by

$$
\tau_{F}=\frac{\left(\begin{array}{c}
3 \\
m+1
\end{array}\right) f_{m, 0}}{r} \text {. }
$$

By such naive multicasting in the fronthaul link, each EN now has access to all the coded messages in (4), and can transmit with full cooperation in the access link. The access channel thus becomes the $\left(\begin{array}{l}3 \\ 3\end{array}\right) \times\left(\begin{array}{c}3 \\ m+1\end{array}\right)$ cooperative X-multicast channel in Definition 11, whose achievable per-user DoF is $d_{m, 3}=1$ in [2, Lemma 1]. Since each UE desires $\left(\begin{array}{c}2 \\ m\end{array}\right)$ coded messages, from Remark 1, the NDT of the access link is given by

$$
\tau_{A}=\frac{\left(\begin{array}{c}
2 \\
m
\end{array}\right) f_{m, 0}}{d_{m, 3}}=\left(\begin{array}{c}
2 \\
m
\end{array}\right) f_{m, 0} .
$$

Summing up (5) and (6), the total NDT for group $(m, 0)$ is

$$
\tau_{m, 0}=\frac{\left(\begin{array}{c}
3 \\
m+1
\end{array}\right) f_{m, 0}}{r}+\left(\begin{array}{c}
2 \\
m
\end{array}\right) f_{m, 0} .
$$

\section{B. Delivery of Group $(m, 1)$}

Unlike the subfiles in group $(m, 0)$, each subfile in group $(m, 1)$ is already cached at one EN, and therefore the coded messages can be generated at each EN locally. In specific, each $\mathrm{EN} p$, for $p \in[3]$, generates:

$$
\left\{W_{\Phi^{+},\{p\}}^{\oplus} \triangleq \bigoplus_{q \in \Phi^{+}} W_{q, \Phi^{+} \backslash\{q\},\{p\}}: \Phi^{+} \subseteq[3],\left|\Phi^{+}\right|=m+1\right\} .
$$

Each coded message $W_{\Phi^{+},\{p\}}^{\oplus}$ has $f_{m, 1} F$ bits, and is desired by UE set $\Phi^{+}$. These coded messages can be delivered to UEs via one hop in the access link without the use of fronthaul link or delivered via two hops with the aid of fronthaul link.

1) Without Fronthaul: Each $\mathrm{EN} p$, for $p \in[3]$, sends $\left\{W_{\Phi^{+},\{p\}}^{\oplus}\right\}$ in the access link, and the access channel becomes the $\left(\begin{array}{l}3 \\ 1\end{array}\right) \times\left(\begin{array}{c}3 \\ m+1\end{array}\right)$ cooperative $\mathrm{X}$-multicast channel with achievable per-user DoF $d_{m, 1}$ in [2, Lemma 1]. Since each UE desires $3\left(\begin{array}{c}2 \\ m\end{array}\right)$ messages, the NDT is given by

$$
\tau=\frac{3\left(\begin{array}{c}
2 \\
m
\end{array}\right) f_{m, 1}}{d_{m, 1}} .
$$

2) With Fronthaul: With the aid of fronthaul, we can allow ENs to access the coded messages of others via the transmission of the MBS in the fronthaul link, thereby enabling transmission cooperation among ENs in the access link. As a price to pay for the EN cooperation gain, additional fronthaul delivery latency is caused. Thus, the optimal cooperation strategy should balance the time between the access link and the fronthaul link.

Assume that after the aid of fronthaul transmission, every set of $1+i$ ENs can form a cooperation group in the access link, where $i \in[2]$ is a design parameter to balance the tradeoff mentioned above. We split each message $W_{\Phi^{+},\{p\}}^{\oplus}$ in (7) into $\left(\begin{array}{l}2 \\ i\end{array}\right)$ sub-messages $\left\{W_{\Phi^{+},\{p\}}^{\oplus, \Psi^{+}}: \Psi^{+} \subseteq[3],\left|\Psi^{+}\right|=1+i, \Psi^{+} \ni p\right\}$, each with $f_{m, 1} /\left(\begin{array}{l}2 \\ i\end{array}\right) F$ bits and sent by EN set $\Psi^{+}$exclusively in the access transmission. Consider an arbitrary EN set $\Psi^{+}$ with size $1+i$. ENs in $\Psi^{+}$need to send sub-messages

$$
\left\{W_{\Phi^{+},\{p\}}^{\oplus, \Psi^{+}}: \Phi^{+} \subseteq[3],\left|\Phi^{+}\right|=m+1, p \in \Psi^{+}\right\}
$$

to UEs, and the MBS needs to send $W_{\Phi^{+},\{p\}}^{\oplus, \Psi^{+}}$to ENs $\left\{p^{\prime}\right.$ : $\left.p^{\prime} \in \Psi^{+} \backslash\{p\}\right\}$ which do not cache it. Given that each submessage is already cached at one EN, coded multicasting can be used in the fronthaul transmission. In specific, the MBS sends coded sub-messages

$\left\{W_{\Phi^{+},\{p\}}^{\oplus, \Psi^{+}} \oplus W_{\Phi^{+},\left\{p^{\prime}\right\}}^{\oplus, \Psi^{+}}: \Phi^{+} \subseteq[3],\left|\Phi^{+}\right|=m+1, p, p^{\prime} \in \Psi^{+}\right\}$ to EN set $\Psi^{+}$. Note that in [9], the MBS sends coded messages generated directly from subfiles $\left\{W_{q, \Phi,\{p\}}\right\}$ to ENs, not from the coded messages in (9). Compared to [9], an additional layer of XOR combining on top of the coded messages desired by UEs is exploited in our scheme. Upon receiving the above coded sub-messages, each $\mathrm{EN}$ in $\Psi^{+}$can decode its desired sub-messages with its local cache. The fronthaul NDT for the given $i$ is thus given by

$$
\tau_{F}=\frac{\left(\begin{array}{c}
3 \\
m+1
\end{array}\right)\left(\begin{array}{c}
3 \\
1+i
\end{array}\right)\left(\begin{array}{c}
1+i \\
2
\end{array}\right) f_{m, 1}}{r\left(\begin{array}{c}
2 \\
i
\end{array}\right)}=\frac{3\left(\begin{array}{c}
3 \\
m+1
\end{array}\right) i f_{m, 1}}{2 r} .
$$

By such coded multicasting in the fronthaul link, each EN set $\Psi^{+}$with size $1+i$ can cooperatively send sub-messages in (9), each desired by $m+1$ UEs. The network in the access transmission is upgraded into the $\left(\begin{array}{c}3 \\ 1+i\end{array}\right) \times\left(\begin{array}{c}3 \\ m+1\end{array}\right)$ cooperative $\mathrm{X}$-multicast channel with achievable per-user DoF $d_{m, 1+i}$ in [2, Lemma 1]. Since each UE wants $\left(\begin{array}{c}2 \\ m\end{array}\right)\left(\begin{array}{c}3 \\ 1+i\end{array}\right)\left(\begin{array}{c}1+i \\ 1\end{array}\right)$ submessages, each with $f_{m, 1} /\left(\begin{array}{l}2 \\ i\end{array}\right) F$ bits, the access NDT is

$$
\tau_{A}=\frac{\left(\begin{array}{c}
2 \\
m
\end{array}\right)\left(\begin{array}{c}
3 \\
1+i
\end{array}\right)\left(\begin{array}{c}
1+i \\
1
\end{array}\right) f_{m, 1}}{\left(\begin{array}{c}
2 \\
i
\end{array}\right) d_{m, 1+i}}=\frac{3\left(\begin{array}{c}
2 \\
m
\end{array}\right) f_{m, 1}}{d_{m, 1+i}} .
$$

Summing up (10) and 11, the total NDT is given by

$$
\tau=\frac{3\left(\begin{array}{c}
3 \\
m+1
\end{array}\right) i f_{m, 1}}{2 r}+\frac{3\left(\begin{array}{c}
2 \\
m
\end{array}\right) f_{m, 1}}{d_{m, 1+i}} .
$$

Choosing the smallest NDT among (8) without fronthaul delivery and (12) with fronthaul delivery for all possible $i$, we obtain the NDT for group $(m, 1)$ as

$$
\tau_{m, 1}=\min _{i \in[2] \cup\{0\}}\left\{\frac{3\left(\begin{array}{c}
3 \\
m+1
\end{array}\right) i f_{m, 1}}{2 r}+\frac{3\left(\begin{array}{c}
2 \\
m
\end{array}\right) f_{m, 1}}{d_{m, 1+i}}\right\} .
$$

Considering all possible $m$ and $n$, the achievable NDT of the $3 \times 3$ Fog-RAN with decentralized caching is $\sum_{m=0}^{2} \sum_{n=0}^{3} \tau_{m, n}$, where $\tau_{m, n}$ is the NDT for group $(m, n)$.

\section{ACHIEVABle UpPeR Bound OF NDT}

Generalizing the achievable scheme in Section III to arbitrary $N_{T}, N_{R} \geq 2$, we obtain an achievable upper bound of the minimum NDT in the Fog-RAN with decentralized cache placement in the following theorem.

Theorem 1 (Achievable NDT). For the cache-aided Fog-RAN with $N_{T} \geq 2 \mathrm{ENs}$, each with a cache of normalized size $\mu_{T}$, $N_{R} \geq 2$ UEs, each with a cache of normalized size $\mu_{R}, N \geq$ $N_{R}$ files, and a wireless fronthaul link with power scaling 
$r>0$, the minimum NDT achieved by decentralized caching is upper bounded by $\tau_{\text {upper }}=\sum_{m=0}^{N_{R}-1} \sum_{n=0}^{N_{T}} \tau_{m, n}$, where

$$
\tau_{m, 0}=\frac{\left(\begin{array}{c}
N_{R} \\
m+1
\end{array}\right) f_{m, 0}}{r}+\frac{\left(\begin{array}{c}
N_{R}-1 \\
m
\end{array}\right) f_{m, 0}}{d_{m, N_{T}}}
$$

and

$$
\tau_{m, n}=\min _{i \in\left[N_{T}-n\right] \cup\{0\}} \tau_{m, n}^{i},
$$

when $n \geq 1$, with

$$
\tau_{m, n}^{i}=\frac{\left(\begin{array}{c}
N_{R} \\
m+1
\end{array}\right)\left(\begin{array}{c}
N_{T} \\
n
\end{array}\right) \min \left\{1, \frac{i}{n+1}\right\} f_{m, n}}{r}+\frac{\left(\begin{array}{c}
N_{R}-1 \\
m
\end{array}\right)\left(\begin{array}{c}
N_{T} \\
n
\end{array}\right) f_{m, n}}{d_{m, n+i}} .
$$

Here $f_{m, n}$ is defined in (1), and $d_{m, j}$ is the achievable peruser DoF of the $\left(\begin{array}{c}N_{T} \\ j\end{array}\right) \times\left(\begin{array}{c}N_{R} \\ m+1\end{array}\right)$ cooperative X-multicast channel given in [2] Lemma 1].

Proof. Similar to Section [III we assume that UE $q$, for $q \in\left[N_{R}\right]$, desires $W_{q}$ in the delivery phase. Excluding the locally cached subfiles, each UE $q$, for $q \in\left[N_{R}\right]$, wants subfiles $\left\{W_{q, \Phi, \Psi}: \Phi \not \supset q, \Phi \subseteq\left[N_{R}\right], \Psi \subseteq\left[N_{T}\right]\right\}$. We divide the subfiles wanted by all UEs into different groups according to the size of $\Phi$ and $\Psi$, indexed by $\{(m, n): 0 \leq m \leq$ $\left.N_{R}-1,0 \leq n \leq N_{T}, m, n \in \mathbb{Z}\right\}$, such that subfiles in group $(m, n)$ are cached at $m$ UEs and $n$ ENs. There are $N_{R}\left(\begin{array}{c}N_{R}-1 \\ m\end{array}\right)\left(\begin{array}{c}N_{T} \\ n\end{array}\right)$ subfiles in group $(m, n)$, each with fractional size $f_{m, n}$. Each group of subfiles is delivered individually in the time division manner. Without loss of generality, we present the delivery strategy for an arbitrary group $(m, n)$. The delivery strategy is also given in Algorithm 1.

1) $n=0$ : Note that each subfile in group $(m, 0)$ is desired by one UE, and already cached at $m$ different UEs but none of ENs. Coded multicasting approach can be used, similar to Section III-A In specific, the coded messages are given by

$$
\left\{W_{\Phi^{+}, \emptyset}^{\oplus} \triangleq \bigoplus_{q \in \Phi^{+}} W_{q, \Phi^{+} \backslash\{q\}, \emptyset}: \Phi^{+} \subseteq\left[N_{R}\right],\left|\Phi^{+}\right|=m+1\right\} .
$$

Each coded message $W_{\Phi^{+}, \emptyset}^{\oplus}$ is desired by UE set $\Phi^{+}$. (If $m=$ 0 , each coded message $W_{\Phi^{+}, \emptyset}^{\oplus}$ degenerates to subfile $W_{q, \emptyset, \emptyset}$ for $\Phi^{+}=\{q\}$.) These messages need to be generated at the MBS and then delivered to UEs via the fronthaul link and the access link. In the fronthaul link, we let the MBS multicast each coded message in (16) to all the $N_{T}$ ENs one by one. The fronthaul NDT is given by

$$
\tau_{F}=\frac{\left(\begin{array}{c}
N_{R} \\
m+1
\end{array}\right) f_{m, 0}}{r} .
$$

By such naive multicast transmission in the fronthaul link, each EN now has access to all the coded messages in (16), and can cooperatively transmit together in the access link. The access channel thus becomes the $\left(\begin{array}{c}N_{T} \\ N_{T}\end{array}\right) \times\left(\begin{array}{c}N_{R} \\ m+1\end{array}\right)$ cooperative $\mathrm{X}$-multicast channel with achievable per-user DoF $d_{m, N_{T}}$ in [2. Lemma 1]. Since each UE desires $\left(\begin{array}{c}N_{R}-1 \\ m\end{array}\right)$ messages, the access NDT is given by

$$
\tau_{A}=\frac{\left(\begin{array}{c}
N_{R}-1 \\
m
\end{array}\right) f_{m, 0}}{d_{m, N_{T}}} .
$$

Combining (17) and (18), the achievable NDT for the delivery

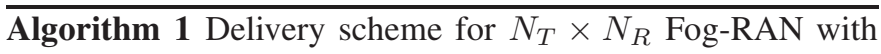
wireless fronthaul

$$
\begin{aligned}
& \text { 1: for } m=0,1, \ldots, N_{R}-1 \text { do } \\
& 2: \quad \text { for } n=0,1, \ldots, N_{T} \text { do } \\
& 3: \quad \text { if } n=0 \text { then }
\end{aligned}
$$

4:

Generate coded messages $\left\{W_{\Phi^{+}, \emptyset}^{\oplus} \triangleq\right.$

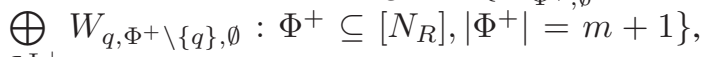
$q \in \Phi^{+}$

each desired by $m+1$ UEs

5: $\quad$ The MBS sends messages $\left\{W_{\Phi^{+}, \emptyset}^{\oplus}\right\}$ to all the $N_{T}$ ENs one by one

6: $\quad$ The network topology in the access link is changed into the $\left(\begin{array}{c}N_{T} \\ N_{T}\end{array}\right) \times\left(\begin{array}{c}N_{R} \\ m+1\end{array}\right)$ cooperative X-multicast channel whose achievable per-user DoF is $d_{m, N_{T}}$ in [2, Lemma 1]
7:

8:

9:

10 :

11:

12 :

13:

14:

15:

17:

18:

19:

20:

21: se

\section{2: $\quad$ end if \\ end for \\ 24: end for}

Generate coded messages $\left\{W_{\Phi^{+}, \Psi}^{\oplus} \triangleq\right.$ $\bigoplus W_{q, \Phi^{+} \backslash\{q\}, \Psi}: \Phi^{+} \subseteq\left[N_{R}\right],\left|\Phi^{+}\right|=$ $q \in \Phi^{+}$

$\left.m+1, \Psi \subseteq\left[N_{T}\right],|\Psi|=n\right\}$

Let $i=\arg \min _{i} \tau_{m, n}^{i}$ in (14)

Split each coded message $W_{\Phi^{+}, \Psi}^{\oplus}$ into $\left(\begin{array}{c}N_{T}-n \\ i\end{array}\right)$ sub-messages $\left\{W_{\Phi^{+}, \Psi}^{\oplus, \Psi^{+}}\right\}$, each with fractional size $\frac{f_{m, n}}{\left({ }^{N_{T}-n}\right)}$ and corresponding to a unique EN set $\Psi^{+}:\left|\Psi^{+}\right|=n+i, \Psi^{+} \supseteq \Psi$

for $\Psi^{+} \subseteq\left[N_{T}\right],\left|\Psi^{+}\right|=n+i$ do

for $\Phi^{+} \subseteq\left[N_{R}\right],\left|\Phi^{+}\right|=m+1$ do

if $\left(\begin{array}{c}n+i \\ n\end{array}\right) \leq\left(\begin{array}{c}n+i \\ n+1\end{array}\right)$ then

The MBS sends sub-messages $\left\{W_{\Phi^{+}, \Psi}^{\oplus, \Psi^{+}}\right.$: $\left.\Psi \subseteq \Psi^{+},|\Psi|=n\right\}$ to EN set $\Psi^{+}$one by one

else

The MBS sends coded sub-messages $\left\{\bigoplus_{\Psi \subset \Psi^{\prime}} W_{\Phi^{+}, \Psi}^{\oplus, \Psi^{+}}: \Psi^{\prime} \subseteq \Psi^{+},\left|\Psi^{\prime}\right|=n+\right.$ $1,|\Psi|=n\}$ to EN set $\Psi^{+}$

\section{end if}

ENs in $\Psi^{+}$can access $\left\{W_{\Phi^{+}, \Psi}^{\oplus, \Psi^{+}}: \Psi \subseteq\right.$ $\left.\Psi^{+},|\Psi|=n\right\}$ desired by UE set $\Phi^{+}$.

\section{end for}

\section{end for}

The network topology in the access link is changed into the $\left(\begin{array}{c}N_{T} \\ n+i\end{array}\right) \times\left(\begin{array}{c}N_{R} \\ m+1\end{array}\right)$ cooperative $\mathrm{X}$-multicast channel whose achievable per-user DoF is $d_{m, n+i}$ in [2, Lemma 1]

if

of group $(m, 0)$ is

$$
\tau_{m, 0}=\frac{\left(\begin{array}{c}
N_{R} \\
m+1
\end{array}\right) f_{m, 0}}{r}+\frac{\left(\begin{array}{c}
N_{R}-1 \\
m
\end{array}\right) f_{m, 0}}{d_{m, N_{T}}} .
$$

2) $n>0$ : Note that each subfile in group $(m, n)$ is desired by one UE, and already cached at $m$ different UEs and 
$n$ different ENs. Coded multicasting approach can be used, similar to Section III-B. In specific, given an arbitrary UE set $\Phi^{+}$with size $\left|\Phi^{+}\right|=m+1$ and an arbitrary EN set $\Psi$ with size $n$, each $\mathrm{EN}$ in $\Psi$ generates the coded message $W_{\Phi^{+}, \Psi}^{\oplus} \triangleq \bigoplus_{q \in \Phi^{+}} W_{q, \Phi^{+} \backslash\{q\}, \Psi}$ desired by all UEs in $\Phi^{+}$. (If $m=0$, coded message $W_{\Phi^{+}, \Psi}^{\oplus}$ degenerates to subfile $W_{q, \emptyset, \Psi}$ for $\Phi^{+}=\{q\}$.) Through this coded multicasting approach, $m+1$ different subfiles are combined into a single coded message via XOR, and there are only $\left(\begin{array}{c}N_{R} \\ m+1\end{array}\right)\left(\begin{array}{c}N_{T} \\ n\end{array}\right)$ coded messages to be transmitted in total, each available at $n$ ENs and desired by $m+1$ UEs.

Similar to Section $\amalg$ II-B, with the aid of fronthaul, we can allow ENs to access the coded messages of others via the transmission of the MBS in the fronthaul link, thereby enabling chances for more transmission cooperation in the access link. Assume that after the aid of fronthaul transmission, every set of $n+i$ ENs can form a cooperation group in the access link, where $i \in\left[N_{T}-n\right] \cup\{0\}$ is a design parameter2 We split each message $W_{\Phi^{+}, \Psi}^{\oplus}$ into $\left(\begin{array}{c}N_{T}-n \\ i\end{array}\right)$ sub-messages, each with fractional size $f_{m, n} /\left(\begin{array}{c}N_{T}-n \\ i\end{array}\right)$ and corresponding to a distinct EN set $\Psi^{+}$with size $n+i$ such that $\Psi^{+} \supseteq \Psi$. Denote $W_{\Phi^{+}, \Psi}^{\oplus, \Psi^{+}}$ as the sub-message in $W_{\Phi^{+}, \Psi}^{\oplus}$, which is desired by UE set $\Phi^{+}$, cached at EN set $\Psi$, and corresponding to EN set $\Psi^{+}$. Each sub-message $W_{\Phi^{+}, \Psi}^{\oplus, \Psi^{+}}$is sent by EN set $\Psi^{+}$exclusively in the access link. Then, for an arbitrary $\mathrm{EN}$ set $\Psi^{+}$with size $n+i$, each $\mathrm{EN}$ in $\Psi^{+}$needs to access all the sub-messages

$$
\left\{W_{\Phi^{+}, \Psi}^{\oplus, \Psi^{+}}: \Phi^{+} \subseteq\left[N_{R}\right],\left|\Phi^{+}\right|=m+1, \Psi \subseteq \Psi^{+},|\Psi|=n\right\} .
$$

To do this, the MBS choose one of the two methods below to send sub-messages to ENs in the fronthaul link.

1) Fronthaul Transmission without Coded Multicasting: For each EN set $\Psi^{+}$, the MBS directly sends submessages in 200 one-by-one, and each EN in $\Psi^{+}$decodes all the non-cached sub-messages. By this method, the NDT in the fronthaul link is given by

$$
\tau_{F}^{1}=\frac{1}{r}\left(\begin{array}{c}
N_{R} \\
m+1
\end{array}\right)\left(\begin{array}{c}
N_{T} \\
n+i
\end{array}\right)\left(\begin{array}{c}
n+i \\
n
\end{array}\right) \frac{f_{m, n}}{\left(\begin{array}{c}
N_{T}-n \\
i
\end{array}\right)} .
$$

2) Fronthaul Transmission with Coded Multicasting: Note that each sub-message is already cached at $n$ ENs. The MBS can exploit coded multicasting opportunities in the fronthaul link. In specific, for each $\mathrm{EN}$ set $\Psi^{+}$, the MBS sends coded sub-messages

$$
\begin{array}{r}
\bigoplus_{\Psi \subset \Psi^{\prime}} W_{\Phi^{+}, \Psi}^{\oplus, \Psi^{+}}: \Phi^{+} \subseteq\left[N_{R}\right],\left|\Phi^{+}\right|=m+1, \Psi^{\prime} \subseteq \Psi^{+}, \\
\left.\left|\Psi^{\prime}\right|=n+1,|\Psi|=n\right\} .
\end{array}
$$

For each coded sub-message $\bigoplus_{\Psi \subset \Psi^{\prime}} W_{\Phi^{+}, \Psi}^{\oplus, \Psi^{+}}$, each EN $p$ in $\Psi^{\prime}$ caches $n$ sub-messages $\left\{W_{\Phi^{+}, \Psi}^{\oplus, \Psi^{+}}: p \in\right.$ $\left.\Psi, \Psi \subset \Psi^{\prime}\right\}$, and can decode the non-cached sub-

\footnotetext{
${ }^{2}$ If $i=0$, every set of $n$ ENs already forms a cooperation group in the access link, and the coded messages can be delivered to UEs directly in the access link without the use of fornthaul link. The access channel becomes the $\left(\begin{array}{c}N_{T} \\ n\end{array}\right) \times\left(\begin{array}{c}N_{R} \\ m+1\end{array}\right)$ cooperative X-multicast channel in [2].
}

message $\left\{W_{\Phi^{+}, \Psi}^{\oplus, \Psi^{+}}: p \notin \Psi, \Psi \subset \Psi^{\prime}\right\}$. By this method the NDT in the fronthaul link is given by

$$
\tau_{F}^{2}=\frac{1}{r}\left(\begin{array}{c}
N_{R} \\
m+1
\end{array}\right)\left(\begin{array}{c}
N_{T} \\
n+i
\end{array}\right)\left(\begin{array}{c}
n+i \\
n+1
\end{array}\right) \frac{f_{m, n}}{\left(\begin{array}{c}
N_{T}-n \\
i
\end{array}\right)} .
$$

Choosing the smaller one between (21) and (22), the fronthaul NDT is given by

$$
\begin{aligned}
\tau_{F} & =\frac{1}{r}\left(\begin{array}{c}
N_{R} \\
m+1
\end{array}\right)\left(\begin{array}{c}
N_{T} \\
n+i
\end{array}\right) \frac{f_{m, n}}{\left(\begin{array}{c}
\left.N_{T}-n\right) \\
i
\end{array}\right)} \min \left\{\left(\begin{array}{c}
n+i \\
n
\end{array}\right),\left(\begin{array}{c}
n+i \\
n+1
\end{array}\right)\right\} \\
& =\left(\begin{array}{c}
N_{R} \\
m+1
\end{array}\right)\left(\begin{array}{c}
N_{T} \\
n
\end{array}\right) \min \left\{1, \frac{i}{n+1}\right\} \frac{f_{m, n}}{r} .
\end{aligned}
$$

Then in the access link, for an arbitrary EN set $\Psi^{+}$with size $n+i$, each $\mathrm{EN}$ in $\Psi^{+}$cooperatively sends sub-messages in (20). The access channel is changed to the $\left(\begin{array}{c}N_{T} \\ n+i\end{array}\right) \times\left(\begin{array}{c}N_{R} \\ m+1\end{array}\right)$ cooperative $\mathrm{X}$-multicast channel with achievable per-user DoF $d_{m, n+i}$ in [2, Lemma 1]. Since each UE $q$, for $q \in\left[N_{R}\right]$, wants $\left(\begin{array}{c}N_{R}-1 \\ m\end{array}\right)\left(\begin{array}{c}N_{T} \\ n+i\end{array}\right)\left(\begin{array}{c}n+i \\ n\end{array}\right)$ sub-messages, the access NDT is

$$
\begin{aligned}
\tau_{A} & =\left(\begin{array}{c}
N_{R}-1 \\
m
\end{array}\right)\left(\begin{array}{c}
N_{T} \\
n+i
\end{array}\right) \frac{\left(\begin{array}{c}
n+i \\
n
\end{array}\right)}{\left(\begin{array}{c}
N_{T}-n \\
i
\end{array}\right)} \frac{f_{m, n}}{d_{m, n+i}} \\
& =\left(\begin{array}{c}
N_{R}-1 \\
m
\end{array}\right)\left(\begin{array}{c}
N_{T} \\
n
\end{array}\right) \frac{f_{m, n}}{d_{m, n+i}} .
\end{aligned}
$$

Combining (23) and 24 and taking the minimum of NDT over $i$, we obtain the NDT for the delivery of group $(m, n)$ as

$$
\tau_{m, n}=\min _{i \in\left[N_{T}-n\right] \cup\{0\}} \tau_{m, n}^{i},
$$

where

$$
\begin{aligned}
\tau_{m, n}^{i}= & \left(\begin{array}{c}
N_{R} \\
m+1
\end{array}\right)\left(\begin{array}{c}
N_{T} \\
n
\end{array}\right) \min \left\{1, \frac{i}{n+1}\right\} \frac{f_{m, n}}{r} \\
& +\left(\begin{array}{c}
N_{R}-1 \\
m
\end{array}\right)\left(\begin{array}{c}
N_{T} \\
n
\end{array}\right) \frac{f_{m, n}}{d_{m, n+i}} .
\end{aligned}
$$

Summing up NDTs in 19 and 25 for all groups, the total achievable NDT is

$$
\tau=\sum_{m=0}^{N_{R}-1} \sum_{n=0}^{N_{T}} \tau_{m, n}
$$

which is the same as in Theorem 1 . Thus, Theorem 1 is proved.

The first and second terms on the right hand side of both (13) and 15 are the fronthaul NDT and the access NDT, respectively. It is clear that the fronthaul NDT decreases as the power scaling $r$ increases. When $r \rightarrow \infty$, the fronthaul NDT approaches zero, and the overall achievable NDT is dominated by the access NDT, given by

$$
\begin{aligned}
\lim _{r \rightarrow \infty} \tau_{\text {upper }} & =\sum_{m=0}^{N_{R}-1} \sum_{n=0}^{N_{T}} \frac{\left(\begin{array}{c}
N_{R}-1 \\
m
\end{array}\right)\left(\begin{array}{c}
N_{T} \\
n
\end{array}\right) f_{m, n}}{d_{m, N_{T}}} \\
& =\sum_{m=0}^{N_{R}-1} \frac{\left(\begin{array}{c}
N_{R}-1 \\
m
\end{array}\right) \mu_{R}^{m}\left(1-\mu_{R}\right)^{N_{R}-m}}{d_{m, N_{T}}}
\end{aligned}
$$

which is equivalent to the NDT when $\mu_{T}=1$. This means that when the fronthaul capacity is large enough, the fronthaul transmission time can be ignored and hence each EN can access the entire database as when $\mu_{T}=1$. The detailed 
discussion of the achievable NDT in Theorem 1 and its comparison to [7], [9]-[11] are given in Section VI

\section{LOWER BOUND OF NDT}

In this section, we present a lower bound of the minimum NDT, based on which we show that the achievable scheme is order-optimal.

Theorem 2 (Lower bound of NDT). For the cache-aided Fog$R A N$ with $N_{T} \geq 2$ ENs, each with a cache of normalized size $\mu_{T}, N_{R} \geq 2$ UEs, each with a cache of normalized size $\mu_{R}$, $N \geq N_{R}$ files, and a wireless fronthaul link with power scaling $r>0$, the minimum NDT achieved by decentralized caching is lower bounded by

$$
\tau_{\text {lower }}=\max _{l_{1} \in\left[N_{R}\right]} \frac{l_{1}\left(1-\mu_{T}\right)^{N_{T}}\left(1-\mu_{R}\right)^{l_{1}}}{r}+\max _{l_{2} \in\left[N_{R}\right]} \frac{l_{2}\left(1-\mu_{R}\right)^{l_{2}}}{\min \left\{l_{2}, N_{T}\right\}} \text {. }
$$

Proof. Since this is the proof of a lower bound, we focus on a specific UE demand that each UE $q\left(q \in\left[N_{R}\right]\right)$ wants file $W_{q}$. Since ENs are assumed to be half-duplex, we will prove the lower bound of fronthaul NDT and access NDT separately.

1) Fronthaul Transmission: We first consider the fronthaul transmission. Consider the transmission of the files desired by the first $l_{1}$ UEs, for $l_{1} \in\left[N_{R}\right]$. The proof is based on the following observation. Given received signals $Q_{1 \sim N_{T}}$ from the MBS at all ENs and the caches $U_{1 \sim N_{T}}$ at all ENs, one can construct the transmitted signals of all ENs. Then, given all the transmitted signals from the ENs and caches $V_{1 \sim l_{1}}$ at the first $l_{1}$ UEs, one can obtain the desired files of these UEs almost surely. We have

$$
H\left(W_{1 \sim l_{1}} \mid Q_{1 \sim N_{T}}, U_{1 \sim N_{T}}, V_{1 \sim l_{1}}\right)=F \varepsilon_{F}+T_{F} \varepsilon_{P} \log P,
$$

where $W_{1 \sim l_{1}}$ are files $\left\{W_{1}, W_{2}, \ldots, W_{l_{1}}\right\}$. Here, $\varepsilon_{F}$ and $\varepsilon_{P}$ are a function of file size $F$ and a function of power $P$, respectively, and satisfy $\lim _{F \rightarrow \infty} \varepsilon_{F}=0, \lim _{P \rightarrow \infty} \varepsilon_{P}=0$. Then, we have

$$
\begin{aligned}
l_{1} F= & H\left(W_{1 \sim l_{1}} \mid W_{l_{1}+1 \sim N}\right) \\
= & I\left(W_{1 \sim l_{1}} ; Q_{1 \sim N_{T}}, U_{1 \sim N_{T}}, V_{1 \sim l_{1}} \mid W_{l_{1}+1 \sim N}\right) \\
& +H\left(W_{1 \sim l_{1}} \mid Q_{1 \sim N_{T}}, U_{1 \sim N_{T}}, V_{1 \sim l_{1}}, W_{l_{1}+1 \sim N}\right) \\
= & H\left(Q_{1 \sim N_{T}}, U_{1 \sim N_{T}}, V_{1 \sim l_{1}} \mid W_{l_{1}+1 \sim N}\right) \\
& -H\left(Q_{1 \sim N_{T}}, U_{1 \sim N_{T}}, V_{1 \sim l_{1}} \mid W_{1 \sim N}\right) \\
& +F \varepsilon_{F}+T_{F} \varepsilon_{P} \log P \\
\leq & H\left(Q_{1 \sim N_{T}}, U_{1 \sim N_{T}}, V_{1 \sim l_{1}} \mid W_{l_{1}+1 \sim N}\right) \\
& +F \varepsilon_{F}+T_{F} \varepsilon_{P} \log P \\
\leq & h\left(Q_{1 \sim N_{T}}\right)+H\left(U_{1 \sim N_{T}}, V_{1 \sim l_{1}} \mid W_{l_{1}+1 \sim N}\right) \\
& +F \varepsilon_{F}+T_{F} \varepsilon_{P} \log P
\end{aligned}
$$

where $W_{l_{1}+1 \sim N}$ are files $\left\{W_{l_{1}+1}, W_{l_{1}+2}, \ldots, W_{N}\right\}$. Here, (27b) and (27c) come from the definition of mutual infor- mation; 27e comes from the fact that conditioning reduces entropy. In 27e,$h\left(Q_{1 \sim N_{T}}\right)$ is bounded by

$$
\begin{aligned}
h\left(Q_{1 \sim N_{T}}\right) & =I\left(Q_{1 \sim N_{T}} ; S\right)+h\left(Q_{1 \sim N_{T}} \mid S\right) \\
& =I\left(Q_{1 \sim N_{T}} ; S\right)+T_{F} \varepsilon_{P} \log P \\
& \leq T_{F}\left(r \log P+\varepsilon_{P} \log P\right)+T_{F} \varepsilon_{P} \log P .
\end{aligned}
$$

Here, $S$ is the transmitted signal of the MBS; 28b is due to the fact that the conditional entropy $h\left(Q_{1 \sim N_{T}} \mid S\right)$ comes from the noise received at ENs; 28c follows from the capacity bound of the broadcast channel in high SNR regime. In 27ed, $H\left(U_{1 \sim N_{T}}, V_{1 \sim l_{1}} \mid W_{l_{1}+1 \sim N}\right)$ is given by

$$
\begin{aligned}
& H\left(U_{1 \sim N_{T}}, V_{1 \sim l_{1}} \mid W_{l_{1}+1 \sim N}\right) \\
= & H\left(U_{1 \sim N_{T}}^{1 \sim l_{1}}, V_{1 \sim l_{1}}^{1 \sim l_{1}}\right) \\
= & \sum_{n=1}^{l_{1}} H\left(U_{1 \sim N_{T}}^{n}, V_{1 \sim l_{1}}^{n}\right) \\
= & l_{1} F \cdot\left[1-\left(1-\mu_{T}\right)^{N_{T}}\left(1-\mu_{R}\right)^{l_{1}}\right] .
\end{aligned}
$$

Here, $U_{1 \sim N_{T}}^{1 \sim l_{1}}, V_{1 \sim l_{1}}^{1 \sim l_{1}}$ are the cached contents of files $\left\{W_{1}, W_{2}, \ldots, W_{l_{1}}\right\}$ at all the $N_{T}$ ENs and UEs $\left\{1,2, \ldots, l_{1}\right\}$, respectively, and $U_{1 \sim N_{T}}^{n}, V_{1 \sim l_{1}}^{n}$ are the cached contents of file $n$ at all the $N_{T}$ ENs and UEs $\left\{1,2, \ldots, l_{1}\right\}$, respectively; (29a) and 29b come from the fact that only the cached contents of files $\left\{W_{1}, \ldots, W_{l_{1}}\right\}$ are unknown given files $\left\{W_{l_{1}+1}, \ldots, W_{N}\right\}$ and that the cache scheme does not allow intra-file coding or inter-file coding; (29c) comes from the fact that each EN and each UE caches a subset of $\mu_{T} F$ and $\mu_{R} F$ bits of each file independently and uniformly at random, respectively.

Combining 27e 28c 29c , and letting $F \rightarrow \infty, P \rightarrow \infty$, we obtain that

$$
\lim _{P \rightarrow \infty} \lim _{F \rightarrow \infty} \frac{T_{F} \log P}{F} \geq \frac{1}{r} l_{1}\left(1-\mu_{T}\right)^{N_{T}}\left(1-\mu_{R}\right)^{l_{1}} .
$$

2) Access Phase: Next we consider the access transmission. The proof method is an extension of the approach in [4, Section VI] by taking decentralized cache scheme into account. Consider the first $l_{2}$ UEs, for $l_{2} \in\left[N_{R}\right]$. The proof is based on the following observation. Given the received signals $Y_{1 \sim l_{2}}$ and the cached contents $V_{1 \sim l_{2}}$ of the $l_{2}$ UEs, one can successfully decode the desired files of these $l_{2}$ UEs. Thus, we have

$$
H\left(W_{1 \sim l_{2}} \mid Y_{1 \sim l_{2}}, V_{1 \sim l_{2}}\right)=F \varepsilon_{F} .
$$

Similar to (27), we have

$$
\begin{aligned}
& l_{2} F=H\left(W_{1 \sim l_{2}} \mid W_{l_{2}+1 \sim N}\right) \\
& =I\left(W_{1 \sim l_{2}} ; Y_{1 \sim l_{2}}, V_{1 \sim l_{2}} \mid W_{l_{2}+1 \sim N}\right) \\
& +H\left(W_{1 \sim l_{2}} \mid Y_{1 \sim l_{2}}, V_{1 \sim l_{2}}, W_{l_{2}+1 \sim N}\right) \\
& =I\left(W_{1 \sim l_{2}} ; Y_{1 \sim l_{2}}, V_{1 \sim l_{2}} \mid W_{l_{2}+1 \sim N}\right)+F \varepsilon_{F} \\
& =H\left(Y_{1 \sim l_{2}}, V_{1 \sim l_{2}} \mid W_{l_{2}+1 \sim N}\right) \\
& -H\left(Y_{1 \sim l_{2}}, V_{1 \sim l_{2}} \mid W_{1 \sim N}\right)+F \varepsilon_{F} \\
& \leq H\left(Y_{1 \sim l_{2}}, V_{1 \sim l_{2}} \mid W_{l_{2}+1 \sim N}\right)+F \varepsilon_{F} \\
& \leq h\left(Y_{1 \sim l_{2}}\right)+H\left(V_{1 \sim l_{2}} \mid W_{l_{2}+1 \sim N}\right)+F \varepsilon_{F} \text {. }
\end{aligned}
$$




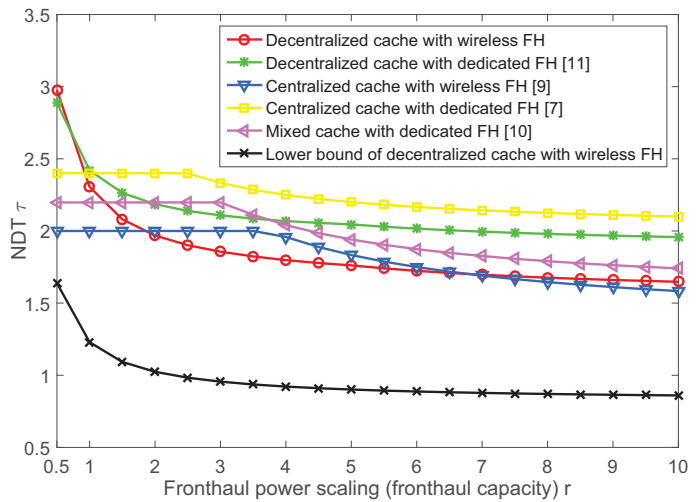

Fig. 2: NDT when $N_{T}=2, N_{R}=5, \mu_{T}=1 / 2, \mu_{R}=1 / 5$.

In (31f), $h\left(Y_{1 \sim l_{2}}\right)$ is bounded by

$$
\begin{aligned}
h\left(Y_{1 \sim l_{2}}\right)= & I\left(Y_{1 \sim l_{2}} ; X_{1 \sim N_{T}}\right)+h\left(Y_{1 \sim l_{2}} \mid X_{1 \sim N_{T}}\right) \\
= & I\left(Y_{1 \sim l_{2}} ; X_{1 \sim N_{T}}\right)+T_{F} \varepsilon_{P} \log P \\
\leq & T_{A} \min \left\{N_{T}, l_{2}\right\}\left(\log P+\varepsilon_{P} \log P\right) \\
& +T_{A} \varepsilon_{P} \log P .
\end{aligned}
$$

Here, $X_{1 \sim N_{T}}$ are the transmitted signals from all the $N_{T}$ ENs; $32 \mathrm{~b}$ is due to the fact that the conditional entropy $h\left(Y_{1 \sim l_{2}} \mid X_{1 \sim N_{T}}\right)$ comes from the noise received at UEs; 32C follows from the capacity bound of the $N_{T} \times l_{2}$ MIMO channel in high SNR regime, similar to the proof of [4, Lemma 5].

In (31f), $H\left(V_{1 \sim l_{2}} \mid W_{l_{2}+1 \sim N}\right)$ is given by

$$
\begin{aligned}
H\left(V_{1 \sim l_{2}} \mid W_{l_{2}+1 \sim N}\right) & =H\left(V_{1 \sim l_{2}}^{1 \sim l_{2}}\right) \\
& =\sum_{n=1}^{l_{2}} H\left(V_{1 \sim l_{2}}^{n}\right) \\
& =l_{2} F \cdot\left[1-\left(1-\mu_{R}\right)^{l_{2}}\right] .
\end{aligned}
$$

Note that (33) is similar to 29], and the detailed explanation is omitted here.

Combining (31f) 32c 33c), and letting $F \rightarrow \infty, P \rightarrow \infty$, we obtain that

$$
\lim _{P \rightarrow \infty} \lim _{F \rightarrow \infty} \frac{T_{A} \log P}{F} \geq \frac{l_{2}\left(1-\mu_{R}\right)^{l_{2}}}{\min \left\{l_{2}, N_{T}\right\}} .
$$

Combining (30) and 34), and taking the maximum over $l_{1}, l_{2} \in\left[N_{R}\right]$, the minimum NDT $\tau$ is lower bounded by

$$
\begin{aligned}
\tau & =\lim _{P \rightarrow \infty} \lim _{F \rightarrow \infty} \frac{\left(T_{F}+T_{A}\right) \log P}{F} \\
& \geq \max _{l_{1} \in\left[N_{R}\right]} \frac{l_{1}}{r}\left(1-\mu_{T}\right)^{N_{T}}\left(1-\mu_{R}\right)^{l_{1}}+\max _{l_{2} \in\left[N_{R}\right]} \frac{l_{2}\left(1-\mu_{R}\right)^{l_{2}}}{\min \left\{l_{2}, N_{T}\right\}},
\end{aligned}
$$

which finishes the proof of Theorem 2 .

Comparing Theorem 1 and Theorem 2, the multiplicative gap between the upper and lower bounds is given in the following corollary, whose proof is in appendix.

Corollary 1 (Gap of NDT). The multiplicative gap between the upper and lower bounds of the minimum NDT of the considered system is within 12.

\section{Numerical Results And Discussions}

In this section, we use numerical examples to compare the achievable NDT of our proposed scheme using decentralized caching with existing schemes, including centralized caching in [73, [9], mixed caching (centralized at ENs and decentralized at UEs) in [10], and decentralized caching (for two ENs only) in [11]. Note that [9] assumed wireless fronthaul, while [7], [10], [11] assumed dedicated fronthaul with capacity of $C_{F}=r \log P$ bits per symbol. Fig. 2 depicts the NDT when $N_{T}=2, N_{R}=5, \mu_{T}=\frac{1}{2}, \mu_{R}=\frac{1}{5}$. It is seen that when $r$ increases, the achievable NDT, as well as our proposed lower bound, decreases and finally approaches a constant as expected.

Comparing to the decentralized caching in [11], it is seen in Fig. 2 that our achievable NDT is better in most fronthaul capacity regions even though dedicated fronthaul link is considered in [11]. This is because EN cooperation in the access link is fully exploited by the careful design of fronthaul transmission in our scheme, while it is only exploited in [11] when transmitting some specific subfiles.

When $r$ is small, it is seen that our scheme with decentralized caching is inferior to the centralized caching in [9] as expected, since [9] can better utilize the cache memory. However, when $r \geq 2$, our scheme performs very close to [9], and even outperforms it when $2 \leq r \leq 6$. This is because 1) an additional layer of coded multicasting opportunities based on the coded messages desired by UEs is exploited in the fronthaul link in our scheme as stated in Section [II-B, while in [9], the coded multicasting opportunities in the fronthaul link are only exploited by generating coded messages directly from requested subfiles $\left\{W_{q, \Phi, \Psi}\right\} ; 2$ ) we obtain a larger achievable per-user DoF than the one in [9] in the access channel by using interference neutralization and interference alignment jointly.

Comparing to the centralized caching in [7] and mixed caching in [10] with dedicated fronthaul, it is seen that our achievable NDT is even better than theirs when $r \geq 2$. This is because in their schemes, the fronthaul link is not used to deliver contents already cached at ENs to boost EN cooperation in the access link. Note that the authors in [8] state that coded multicasting is not useful in certain cases in the fronthaul delivery. However, by comparing to [7] with conventional uncoded caching at UEs, it is still seen that coded multicasting plays an important role in the access delivery in our scheme to reduce the sum NDT.

\section{CONCLUSION}

In this paper, we investigated the latency performance of a Fog-RAN with wireless fronthaul and for arbitrary number of cache-equipped ENs and cache-equipped UEs, by using NDT as the performance metric. The system consists of two phases: a file-splitting based decentralized cache placement phase and a fronthaul-aided two-hop content delivery phase. In our proposed delivery scheme, coded multicasting opportunities

\footnotetext{
${ }^{3}$ Since [7] only considers EN caches, we add conventional uncoded caching at UEs in the plot of [7] for fair comparison.
} 
are fully exploited in the fronthaul link by fetching both noncached and cached contents of ENs to enhance EN cooperation in the access transmission. Then, the access link is changed into the cooperative X-multicast channels. We obtained both the achievable upper bound and theoretical lower bound of NDT for decentralized caching, with a multiplicative gap less than 12. It is shown that our decentralized caching scheme can balance the time between the fronthaul link and access link by the careful design of fronthaul transmission, and even outperforms the centralized schemes, mixed scheme, and decentralized scheme (with dedicated fronthaul) under certain conditions.

\section{APPENDIX: PROOF OF COROLlary 1}

In Appendix, we aim to prove Corollary 1. We consider two cases to prove the gap, i.e., $N_{T} \geq N_{R}$ and $N_{T}<N_{R}$.

\section{A. $N_{T} \geq N_{R}$}

We first consider the case when $N_{T} \geq N_{R}$. The achievable upper bound of NDT is $\tau_{\text {upper }}=\sum_{m=0}^{N_{R}-1} \sum_{n=0}^{N_{T}} \tau_{m, n}$, where $\tau_{m, n}$ is given in (13) and (14). Taking $i=0$ in (14), $\tau_{m, n}$ $(n>0)$ is bounded by

$$
\tau_{m, n} \leq \frac{\left(\begin{array}{c}
N_{R}-1 \\
m
\end{array}\right)\left(\begin{array}{c}
N_{T} \\
n
\end{array}\right) f_{m, n}}{d_{m, n}}
$$

We also have

$$
\tau_{m, 0}=\left(\begin{array}{c}
N_{R} \\
m+1
\end{array}\right) \frac{f_{m, 0}}{r}+\frac{\left(\begin{array}{c}
N_{R}-1 \\
m
\end{array}\right) f_{m, 0}}{d_{m, N_{T}}} .
$$

When $N_{T} \geq N_{R}$, it is easy to see that $d_{m, n} \geq 1 / 2$ for $m \in$ $\left[N_{R}-1\right] \cup\{0\}, n \in\left[N_{T}\right]$. Then, $\tau_{\text {upper }}$ is upper bounded by (35). Taking $l_{2}=1$ in (26), the lower bound of NDT is lower bounded by

$$
\tau_{\text {lower }} \geq \max _{l_{1}} \frac{l_{1}\left(1-\mu_{T}\right)^{N_{T}}\left(1-\mu_{R}\right)^{l_{1}}}{r}+\left(1-\mu_{R}\right) .
$$

Denote $g$ as the multiplicative gap, then the gap is bounded by

$$
g \leq \frac{2\left(1-\mu_{R}\right)+\frac{\left(1-\mu_{T}\right)^{N_{T}}}{r} \frac{1-\mu_{R}}{\mu_{R}}\left[1-\left(1-\mu_{R}\right)^{N_{R}}\right]}{\max _{l_{1}} \frac{l_{1}\left(1-\mu_{T}\right)^{N_{T}}\left(1-\mu_{R}\right)^{l_{1}}}{r}+\left(1-\mu_{R}\right)} .
$$

To upper bound $g$, we first consider

$$
\begin{aligned}
g_{F} & \triangleq \frac{\frac{\left(1-\mu_{T}\right)^{N_{T}}}{r} \frac{1-\mu_{R}}{\mu_{R}}\left[1-\left(1-\mu_{R}\right)^{N_{R}}\right]}{\max _{l_{1}} \frac{l_{1}\left(1-\mu_{T}\right)^{N_{T}}\left(1-\mu_{R}\right)^{l_{1}}}{r}} \\
& =\frac{\frac{1-\mu_{R}}{\mu_{R}}\left[1-\left(1-\mu_{R}\right)^{N_{R}}\right]}{\max _{l_{1}} l_{1}\left(1-\mu_{R}\right)^{l_{1}}},
\end{aligned}
$$

which can also be viewed as the gap in the fronthaul link. We consider four cases to upper bound $g_{F}$, i.e. (1) $N_{R} \leq 12$; (2) $N_{R} \geq 13, \mu_{R} \geq \frac{1}{12}$; (3) $N_{R} \geq 13, \frac{1}{N_{R}} \leq \mu_{R}<\frac{1}{12}$; (4) $N_{R} \geq 13, \mu_{R}<\frac{1}{N_{R}}$. Note that the broadcast channel in the fronthaul link is similar to the one-server shared link in [1], [6], and the proof here is similar to the one in [1], [6].

1) $N_{R} \leq 12$ : In this case, using the inequality (1 $\left.\mu_{R}\right)^{N_{R}} \geq 1-N_{R} \mu_{R}$, we have

$\frac{1-\mu_{R}}{\mu_{R}}\left[1-\left(1-\mu_{R}\right)^{N_{R}}\right] \leq \frac{1-\mu_{R}}{\mu_{R}} N_{R} \mu_{R} \leq 12\left(1-\mu_{R}\right)$. Letting $l_{1}=1, g_{F}$ is bounded by

$$
g_{F} \leq \frac{12\left(1-\mu_{R}\right)}{1-\mu_{R}}=12 .
$$

2) $N_{R} \geq 13, \mu_{R} \geq \frac{1}{12}$ : We have

$$
\frac{1-\mu_{R}}{\mu_{R}}\left[1-\left(1-\mu_{R}\right)^{N_{R}}\right] \leq \frac{1-\mu_{R}}{\mu_{R}} \leq 12\left(1-\mu_{R}\right) \text {. }
$$

Similar to Case 1 that $N_{R} \leq 12, g_{F}$ is also upper bounded by 12. 


$$
\begin{aligned}
\tau_{\text {upper }} & \leq \sum_{m=0}^{N_{R}-1} \sum_{n=1}^{N_{T}} \frac{\left(\begin{array}{c}
N_{R}-1 \\
m
\end{array}\right)\left(\begin{array}{c}
N_{T} \\
n
\end{array}\right) f_{m, n}}{d_{m, n}}+\sum_{m=0}^{N_{R}-1} \frac{\left(\begin{array}{c}
N_{R}-1 \\
m
\end{array}\right) f_{m, 0}}{d_{m, N_{T}}}+\frac{1}{r} \sum_{m=0}^{N_{R}-1}\left(\begin{array}{c}
N_{R} \\
m+1
\end{array}\right) f_{m, 0} \\
& \leq 2 \sum_{m=0}^{N_{R}-1} \sum_{n=0}^{N_{T}}\left(\begin{array}{c}
N_{R}-1 \\
m
\end{array}\right)\left(\begin{array}{c}
N_{T} \\
n
\end{array}\right) f_{m, n}+\frac{\left(1-\mu_{T}\right)^{N_{T}}}{r} \sum_{m=0}^{N_{R}-1}\left(\begin{array}{c}
N_{R} \\
m+1
\end{array}\right) \mu_{R}^{m}\left(1-\mu_{R}\right)^{N_{R}-m} \\
& =2\left(1-\mu_{R}\right)+\frac{\left(1-\mu_{T}\right)^{N_{T}}}{r} \frac{1-\mu_{R}}{\mu_{R}} \sum_{m=0}^{N_{R}-1}\left(\begin{array}{c}
N_{R} \\
m+1
\end{array}\right) \mu_{R}^{m+1}\left(1-\mu_{R}\right)^{N_{R}-m-1} \\
& =2\left(1-\mu_{R}\right)+\frac{\left(1-\mu_{T}\right)^{N_{T}}}{r} \frac{1-\mu_{R}}{\mu_{R}} \sum_{p=1}^{N_{R}}\left(\begin{array}{c}
N_{R} \\
p
\end{array}\right) \mu_{R}^{p}\left(1-\mu_{R}\right)^{N_{R}-p} \\
& =2\left(1-\mu_{R}\right)+\frac{\left(1-\mu_{T}\right)^{N_{T}}}{r} \frac{1-\mu_{R}}{\mu_{R}}\left[\sum_{p=0}^{N_{R}}\left(\begin{array}{c}
N_{R} \\
p
\end{array}\right) \mu_{R}^{p}\left(1-\mu_{R}\right)^{N_{R}-p}-\left(1-\mu_{R}\right)^{N_{R}}\right] \\
& =2\left(1-\mu_{R}\right)+\frac{\left(1-\mu_{T}\right)^{N_{T}}}{r} \frac{1-\mu_{R}}{\mu_{R}}\left[1-\left(1-\mu_{R}\right)^{N_{R}}\right]
\end{aligned}
$$

3) $N_{R} \geq 13, \frac{1}{N_{R}} \leq \mu_{R}<\frac{1}{12}$ : Letting $l_{1}=\left\lfloor\frac{1}{4 \mu_{R}}\right\rfloor$, we have

$$
\begin{aligned}
\max _{l_{1}} l_{1}\left(1-\mu_{R}\right)^{l_{1}} & \geq\left\lfloor\frac{1}{4 \mu_{R}}\right\rfloor\left(1-\mu_{R}\right)^{\left\lfloor\frac{1}{4 \mu_{R}}\right\rfloor} \\
& \geq\left\lfloor\frac{1}{4 \mu_{R}}\right\rfloor\left(1-\left\lfloor\frac{1}{4 \mu_{R}}\right\rfloor \mu_{R}\right) \\
& \geq\left(\frac{1}{4 \mu_{R}}-1\right)\left(1-\frac{1}{4 \mu_{R}} \mu_{R}\right) \\
& =\frac{3}{16 \mu_{R}}-\frac{3}{4} .
\end{aligned}
$$

Then, $g_{F}$ is upper bounded by

$$
\begin{aligned}
g_{F} & \leq \frac{\frac{1-\mu_{R}}{\mu_{R}}\left[1-\left(1-\mu_{R}\right)^{N_{R}}\right]}{\frac{3}{16 \mu_{R}}-\frac{3}{4}} \\
& \leq \frac{1 / \mu_{R}}{\frac{3}{16 \mu_{R}}-\frac{3}{4}} \\
& =\frac{1}{3 / 16-3 \mu_{R} / 4} \\
& <\frac{1}{3 / 16-3 / 48}=8 .
\end{aligned}
$$

4) $N_{R} \geq 13, \mu_{R}<\frac{1}{N_{R}}$ : Letting $l_{1}=\left\lfloor\frac{N_{R}}{4}\right\rfloor$, we have

$$
\begin{aligned}
g_{F} & \leq \frac{\frac{1-\mu_{R}}{\mu_{R}}\left[1-\left(1-\mu_{R}\right)^{N_{R}}\right]}{\left\lfloor\frac{N_{R}}{4}\right\rfloor\left(1-\mu_{R}\right)^{\left\lfloor\frac{N_{R}}{4}\right\rfloor}} \\
& =\frac{1-\left(1-\mu_{R}\right)^{N_{R}}}{\mu_{R}\left\lfloor\frac{N_{R}}{4}\right\rfloor\left(1-\mu_{R}\right)^{\left\lfloor\frac{N_{R}}{4}\right\rfloor-1}} \\
& \leq \frac{1-\left(1-N_{R} \mu_{R}\right)}{\mu_{R}\left\lfloor\frac{N_{R}}{4}\right\rfloor\left(1-\mu_{R}\right)^{\left\lfloor\frac{N_{R}}{4}\right\rfloor-1}} \\
& =\frac{N_{R}}{\left\lfloor\frac{N_{R}}{4}\right\rfloor} \frac{1}{\left(1-\mu_{R}\right)^{\left\lfloor\frac{N_{R}}{4}\right\rfloor-1}} \\
& \leq \frac{N_{R}}{\left\lfloor\frac{N_{R}}{4}\right\rfloor} \frac{1}{1-\left(\left\lfloor\frac{N_{R}}{4}\right\rfloor-1\right) \mu_{R}} \\
& \leq \frac{N_{R}}{\frac{N_{R}}{4}-1} \frac{1}{1-\left(\frac{N_{R}}{4}-1\right) \mu_{R}} \\
& <\frac{1}{\frac{1}{4}-\frac{1}{N_{R}}} \frac{1}{1-\left(\frac{N_{R}}{4}-1\right) \frac{1}{N_{R}}} \\
& \leq \frac{1}{\frac{1}{4}-\frac{1}{13}} \frac{1}{\frac{3}{4}+\frac{1}{N_{R}}}<8 .
\end{aligned}
$$

Combining all four cases, we find that $g_{F} \leq 12$ for all $\mu_{R}, N_{R}$. Then, the gap $g$ is upper bounded by

$$
g \leq \frac{2\left(1-\mu_{R}\right)+12 \max _{l_{1}} \frac{l_{1}\left(1-\mu_{T}\right)^{N_{T}}\left(1-\mu_{R}\right)^{l_{1}}}{r}}{\max _{l_{1}} \frac{l_{1}\left(1-\mu_{T}\right)^{N_{T}}\left(1-\mu_{R}\right)^{l_{1}}}{r}+\left(1-\mu_{R}\right)} \leq 12 .
$$

Thus, we proved the case when $N_{T} \geq N_{R}$.

B. $N_{T}<N_{R}$

Now, we consider the case when $N_{T}<N_{R}$. The achievable upper bound of NDT is $\tau_{\text {upper }}=\sum_{m=0}^{N_{R}-1} \sum_{n=0}^{N_{T}} \tau_{m, n}$, where $\tau_{m, n}$ is given in (13) and (14). Taking $i=0$ in (14), $\tau_{m, n}$ $(n>0)$ is bounded by

$$
\tau_{m, n} \leq \frac{\left(\begin{array}{c}
N_{R}-1 \\
m
\end{array}\right)\left(\begin{array}{c}
N_{T} \\
n
\end{array}\right) f_{m, n}}{d_{m, n}} .
$$




$$
\begin{aligned}
& \tau_{\text {upper }} \leq \sum_{m=0}^{N_{R}-1} \sum_{n=1}^{N_{T}} \frac{\left(\begin{array}{c}
N_{R}-1 \\
m
\end{array}\right)\left(\begin{array}{c}
N_{T} \\
n
\end{array}\right) f_{m, n}}{d_{m, n}}+\sum_{m=0}^{N_{R}-1} \frac{\left(\begin{array}{c}
N_{R}-1 \\
m
\end{array}\right) f_{m, 0}}{d_{m, N_{T}}}+\frac{1}{r} \sum_{m=0}^{N_{R}-1}\left(\begin{array}{c}
N_{R} \\
m+1
\end{array}\right) f_{m, 0} \\
& \leq \sum_{m=0}^{N_{R}-1} \sum_{n=1}^{N_{T}} \frac{\left(\begin{array}{c}
N_{R}-1 \\
m
\end{array}\right)\left(\begin{array}{c}
N_{T} \\
n
\end{array}\right) f_{m, n}}{\frac{N_{T}}{N_{T}+\frac{N_{R}-m-1}{m+1}}}+\sum_{m=0}^{N_{R}-1} \frac{\left(\begin{array}{c}
N_{R}-1 \\
m
\end{array}\right) f_{m, 0}}{\frac{N_{T}}{N_{T}+\frac{N_{R}-m-1}{m+1}}}+\frac{1}{r} \sum_{m=0}^{N_{R}-1}\left(\begin{array}{c}
N_{R} \\
m+1
\end{array}\right) f_{m, 0} \\
& =\sum_{m=0}^{N_{R}-1} \frac{\left(\begin{array}{c}
N_{R}-1 \\
m
\end{array}\right)}{\frac{N_{T}}{N_{T}+\frac{N_{R}-m-1}{m+1}}} \sum_{n=0}^{N_{T}}\left(\begin{array}{c}
N_{T} \\
n
\end{array}\right) f_{m, n}+\frac{1}{r} \sum_{m=0}^{N_{R}-1}\left(\begin{array}{c}
N_{R} \\
m+1
\end{array}\right) f_{m, 0} \\
& =\sum_{m=0}^{N_{R}-1} \frac{\left(\begin{array}{c}
N_{R}-1 \\
m
\end{array}\right)}{\frac{N_{T}}{N_{T}+\frac{N_{R}-m-1}{m+1}}} \mu_{R}^{m}\left(1-\mu_{R}\right)^{N_{R}-m}+\frac{1}{r} \sum_{m=0}^{N_{R}-1}\left(\begin{array}{c}
N_{R} \\
m+1
\end{array}\right) f_{m, 0} \\
& =\frac{N_{T}-1}{N_{T}} \sum_{m=0}^{N_{R}-1}\left(\begin{array}{c}
N_{R}-1 \\
m
\end{array}\right) \mu_{R}^{m}\left(1-\mu_{R}\right)^{N_{R}-m}+\frac{1}{N_{T}} \sum_{m=0}^{N_{R}-1}\left(\begin{array}{c}
N_{R} \\
m+1
\end{array}\right) \mu_{R}^{m}\left(1-\mu_{R}\right)^{N_{R}-m}+\frac{1}{r} \sum_{m=0}^{N_{R}-1}\left(\begin{array}{c}
N_{R} \\
m+1
\end{array}\right) f_{m, 0} \\
& =\frac{N_{T}-1}{N_{T}}\left(1-\mu_{R}\right) \sum_{m=0}^{N_{R}-1}\left(\begin{array}{c}
N_{R}-1 \\
m
\end{array}\right) \mu_{R}^{m}\left(1-\mu_{R}\right)^{N_{R}-m-1}+\frac{1-\mu_{R}}{N_{T} \mu_{R}} \sum_{m=0}^{N_{R}-1}\left(\begin{array}{c}
N_{R} \\
m+1
\end{array}\right) \mu_{R}^{m+1}\left(1-\mu_{R}\right)^{N_{R}-m-1} \\
& +\frac{1}{r} \sum_{m=0}^{N_{R}-1}\left(\begin{array}{c}
N_{R} \\
m+1
\end{array}\right) f_{m, 0} \\
& =\frac{N_{T}-1}{N_{T}}\left(1-\mu_{R}\right)+\frac{1-\mu_{R}}{N_{T} \mu_{R}} \sum_{p=1}^{N_{R}}\left(\begin{array}{c}
N_{R} \\
p
\end{array}\right) \mu_{R}^{p}\left(1-\mu_{R}\right)^{N_{R}-p}+\frac{1}{r} \sum_{m=0}^{N_{R}-1}\left(\begin{array}{c}
N_{R} \\
m+1
\end{array}\right) f_{m, 0} \\
& =\frac{N_{T}-1}{N_{T}}\left(1-\mu_{R}\right)+\frac{1-\mu_{R}}{N_{T} \mu_{R}}\left[\sum_{p=0}^{N_{R}}\left(\begin{array}{c}
N_{R} \\
p
\end{array}\right) \mu_{R}^{p}\left(1-\mu_{R}\right)^{N_{R}-p}-\left(1-\mu_{R}\right)^{N_{R}}\right]+\frac{1}{r} \sum_{m=0}^{N_{R}-1}\left(\begin{array}{c}
N_{R} \\
m+1
\end{array}\right) f_{m, 0} \\
& =\frac{N_{T}-1}{N_{T}}\left(1-\mu_{R}\right)+\frac{1-\mu_{R}}{N_{T} \mu_{R}}\left[1-\left(1-\mu_{R}\right)^{N_{R}}\right]+\frac{\left(1-\mu_{T}\right)^{N_{T}}}{r} \frac{1-\mu_{R}}{\mu_{R}}\left[1-\left(1-\mu_{R}\right)^{N_{R}}\right]
\end{aligned}
$$

$$
g \leq \frac{\frac{N_{T}-1}{N_{T}}\left(1-\mu_{R}\right)+\frac{1-\mu_{R}}{N_{T} \mu_{R}}\left[1-\left(1-\mu_{R}\right)^{N_{R}}\right]+\frac{\left(1-\mu_{T}\right)^{N_{T}}}{r} \frac{1-\mu_{R}}{\mu_{R}}\left[1-\left(1-\mu_{R}\right)^{N_{R}}\right]}{\max _{l_{1} \in\left[N_{R}\right]} \frac{l_{1}\left(1-\mu_{T}\right)^{N_{T}}\left(1-\mu_{R}\right)^{l_{1}}}{r}+\max _{l_{2} \in\left[N_{R}\right]} \frac{l_{2}\left(1-\mu_{R}\right)^{l_{2}}}{\min \left\{l_{2}, N_{T}\right\}}}
$$

We also have

$$
\tau_{m, 0}=\left(\begin{array}{c}
N_{R} \\
m+1
\end{array}\right) \frac{f_{m, 0}}{r}+\frac{\left(\begin{array}{c}
N_{R}-1 \\
m
\end{array}\right) f_{m, 0}}{d_{m, N_{T}}} .
$$

It is easy to see in [2, Lemma 1] that $d_{m, n} \geq d_{m, 1}=$ $\frac{N_{T}}{N_{T}+\frac{N_{R}-m-1}{m+1}}$ for $m \in\left[N_{R}-1\right] \cup\{0\}, n \in\left[N_{T}\right]$. Then, the achievable upper bound of NDT is bounded by (37). Using Theorem 22, the multiplicative gap $g$ is bounded by (38). In (38), from the analysis when $N_{T} \geq N_{R}$, we have

$$
\frac{\frac{\left(1-\mu_{T}\right)^{N_{T}}}{r} \frac{1-\mu_{R}}{\mu_{R}}\left[1-\left(1-\mu_{R}\right)^{N_{R}}\right]}{\max _{l_{1} \in\left[N_{R}\right]} \frac{l_{1}\left(1-\mu_{T}\right)^{N_{T}}\left(1-\mu_{R}\right)^{l_{1}}}{r}} \leq 12 .
$$

Then, to bound $g$ in (38), we first consider

$$
g_{A} \triangleq \frac{\frac{N_{T}-1}{N_{T}}\left(1-\mu_{R}\right)+\frac{1-\mu_{R}}{N_{T} \mu_{R}}\left[1-\left(1-\mu_{R}\right)^{N_{R}}\right]}{\max _{l_{2} \in\left[N_{R}\right]} \frac{l_{2}\left(1-\mu_{R}\right)^{l_{2}}}{\min \left\{l_{2}, N_{T}\right\}}},
$$

which can also be viewed as the multiplicative gap in the access link. We use three cases to upper bound $g_{A}$, i.e., (1) $\mu_{R}<\frac{1}{4 N_{R}}$; (2) $\frac{1}{4 N_{R}} \leq \mu_{R}<\frac{1}{4 N_{T}}$; (3) $\mu_{R} \geq \frac{1}{4 N_{T}}$.
1) $\mu_{R}<\frac{1}{4 N_{R}}$ : Letting $l_{2}=N_{R}$, we have

$$
\begin{aligned}
\max _{l_{2} \in\left[N_{R}\right]} \frac{l_{2}\left(1-\mu_{R}\right)^{l_{2}}}{\min \left\{l_{2}, N_{T}\right\}} & \geq \frac{N_{R}\left(1-\mu_{R}\right)^{N_{R}}}{N_{T}} \\
& \geq \frac{N_{R}\left(1-N_{R} \mu_{R}\right)}{N_{T}} \\
& >\frac{N_{R}}{N_{T}}\left(1-N_{R} \frac{1}{4 N_{R}}\right)=\frac{3 N_{R}}{4 N_{T}} .
\end{aligned}
$$

Letting $l_{2}=1$, we have

$$
\max _{l_{2} \in\left[N_{R}\right]} \frac{l_{2}\left(1-\mu_{R}\right)^{l_{2}}}{\min \left\{l_{2}, N_{T}\right\}} \geq 1-\mu_{R} .
$$

We also have

$$
\begin{aligned}
\frac{1-\mu_{R}}{N_{T} \mu_{R}}\left[1-\left(1-\mu_{R}\right)^{N_{R}}\right] & \leq \frac{1-\mu_{R}}{N_{T} \mu_{R}}\left[1-\left(1-N_{R} \mu_{R}\right)\right] \\
& =\frac{N_{R}\left(1-\mu_{R}\right)}{N_{T}} \\
& \leq \frac{N_{R}}{N_{T}}
\end{aligned}
$$


Combining 39, 40, 411, $g_{A}$ is upper bounded by

$$
\begin{aligned}
g_{A} & =\frac{\frac{N_{T}-1}{N_{T}}\left(1-\mu_{R}\right)+\frac{1-\mu_{R}}{N_{T} \mu_{R}}\left[1-\left(1-\mu_{R}\right)^{N_{R}}\right]}{\max _{l_{2}} \frac{l_{2}\left(1-\mu_{R}\right)^{l_{2}}}{\min \left\{l_{2}, N_{T}\right\}}} \\
& \leq \frac{\frac{N_{T}-1}{N_{T}}\left(1-\mu_{R}\right)}{1-\mu_{R}}+\frac{\frac{N_{R}}{N_{T}}}{\frac{3 N_{R}}{4 N_{T}}} \\
& <1+4 / 3=7 / 3 .
\end{aligned}
$$

2) $\frac{1}{4 N_{R}} \leq \mu_{R}<\frac{1}{4 N_{T}}$ : Letting $l_{2}=\left\lceil\frac{1}{4 \mu_{R}}\right\rceil$, we have

$$
\begin{aligned}
& \max _{l_{2}} \frac{l_{2}\left(1-\mu_{R}\right)^{l_{2}}}{\min \left\{l_{2}, N_{T}\right\}} \\
\geq & \frac{\left\lceil\frac{1}{4 \mu_{R}}\right\rceil\left(1-\mu_{R}\right)^{\left\lceil\frac{1}{4 \mu_{R}}\right\rceil}}{\min \left\{\left\lceil\frac{1}{4 \mu_{R}}\right\rceil, N_{T}\right\}} \\
\geq & \frac{\frac{1}{4 \mu_{R}}\left(1-\left\lceil\frac{1}{4 \mu_{R}}\right\rceil \mu_{R}\right)}{N_{T}} \\
\geq & \frac{1-\left(\frac{1}{4 \mu_{R}}+1\right) \mu_{R}}{4 \mu_{R} N_{T}} \\
= & \frac{\frac{3}{4}-\mu_{R}}{4 N_{T} \mu_{R}} \\
> & \frac{\frac{3}{4}-\frac{1}{8}}{4 N_{T} \mu_{R}}=\frac{5}{32 N_{T} \mu_{R}} .
\end{aligned}
$$

We also have

$$
\frac{1-\mu_{R}}{N_{T} \mu_{R}}\left[1-\left(1-\mu_{R}\right)^{N_{R}}\right] \leq \frac{1}{N_{T} \mu_{R}} .
$$

Combining (40) (42) (43), $g_{A}$ is upper bounded by

$$
\begin{aligned}
g_{A} & =\frac{\frac{N_{T}-1}{N_{T}}\left(1-\mu_{R}\right)+\frac{1-\mu_{R}}{N_{T} \mu_{R}}\left[1-\left(1-\mu_{R}\right)^{N_{R}}\right]}{\max _{l_{2}} \frac{l_{2}\left(1-\mu_{R}\right)^{l_{2}}}{\min \left\{l_{2}, N_{T}\right\}}} \\
& \leq \frac{\frac{N_{T}-1}{N_{T}}\left(1-\mu_{R}\right)}{1-\mu_{R}}+\frac{\frac{1}{N_{T} \mu_{R}}}{\frac{5}{32 N_{T} \mu_{R}}} \\
& <1+32 / 5=37 / 5 .
\end{aligned}
$$

3) $\mu_{R} \geq \frac{1}{4 N_{T}}$ : Letting $l_{2}=\left\lfloor\frac{1}{4 \mu_{R}}\right\rfloor$, we have

$$
\begin{aligned}
& \max _{l_{2}} \frac{l_{2}\left(1-\mu_{R}\right)^{l_{2}}}{\min \left\{l_{2}, N_{T}\right\}} \\
\geq & \frac{\left.\left\lfloor\frac{1}{4 \mu_{R}}\right\rfloor\left(1-\mu_{R}\right)^{\frac{1}{4 \mu_{R}}}\right\rfloor}{\min \left\{\left\lfloor\frac{1}{4 \mu_{R}}\right\rfloor, N_{T}\right\}} \\
= & \left(1-\mu_{R}\right)^{\left\lfloor\frac{1}{4 \mu_{R}}\right\rfloor} \\
\geq & 1-\left\lfloor\frac{1}{4 \mu_{R}}\right\rfloor \mu_{R} \\
\geq & 1-\frac{1}{4 \mu_{R}} \mu_{R}=\frac{3}{4} .
\end{aligned}
$$

We also have

$$
\frac{1-\mu_{R}}{N_{T} \mu_{R}}\left[1-\left(1-\mu_{R}\right)^{N_{R}}\right] \leq \frac{1}{N_{T} \mu_{R}} \leq \frac{1}{N_{T} \frac{1}{4 N_{T}}}=4 .
$$

Combining (40) (44) (45), $g_{A}$ is bounded by

$$
\begin{aligned}
g_{A} & =\frac{\frac{N_{T}-1}{N_{T}}\left(1-\mu_{R}\right)+\frac{1-\mu_{R}}{N_{T} \mu_{R}}\left[1-\left(1-\mu_{R}\right)^{N_{R}}\right]}{\max _{l_{2}} \frac{l_{2}\left(1-\mu_{R}\right)^{l_{2}}}{\min \left\{l_{2}, N_{T}\right\}}} \\
& \leq \frac{\frac{N_{T}-1}{N_{T}}\left(1-\mu_{R}\right)}{1-\mu_{R}}+\frac{4}{3 / 4} \\
& <1+16 / 3=19 / 3 .
\end{aligned}
$$

From the above three cases, we find that $g_{A}<12$. Then the multiplicative gap $g$ is bounded by (46), when $N_{T}<N_{R}$.

Thus we finished the proof of Corollary 11 that the multiplicative gap is within 12 .

\section{REFERENCES}

[1] M. A. Maddah-Ali and U. Niesen, "Fundamental limits of caching," IEEE Trans. on Infor. Theory, vol. 60, no. 5, pp. 2856-2867, May 2014.

[2] F. Xu, M. Tao, and K. Liu, "Fundamental tradeoff between storage and latency in cache-aided wireless interference networks," IEEE Trans. on Infor. Theory, vol. 63, no. 11, pp. 7464-7491, Nov 2017.

[3] N. Naderializadeh, M. A. Maddah-Ali, and A. S. Avestimehr, "Fundamental limits of cache-aided interference management," IEEE Trans. on Infor. Theory, vol. 63, no. 5, pp. 3092-3107, May 2017.

[4] J. Hachem, U. Niesen, and S. Diggavi, "Degrees of freedom of cacheaided wireless interference networks," to appear in IEEE Trans. on Infor. Theory, 2018, DOI: 10.1109/TIT.2018.2825321.

[5] J. S. P. Roig, D. Gündüz, and F. Tosato, "Interference networks with caches at both ends," in ICC, May 2017.

[6] M. A. Maddah-Ali and U. Niesen, "Decentralized coded caching attains order-optimal memory-rate tradeoff," IEEE/ACM Trans. on Networking, vol. 23, no. 4, pp. 1029-1040, Aug 2015.

[7] A. Sengupta, R. Tandon, and O. Simeone, "Fog-aided wireless networks for content delivery: Fundamental latency tradeoffs," IEEE Trans. on Infor. Theory, vol. 63, no. 10, pp. 6650-6678, Oct 2017.

[8] J. Koh, O. Simeone, R. Tandon, and J. Kang, "Cloud-aided edge caching with wireless multicast fronthauling in fog radio access networks," in WCNC, March 2017.

[9] T. Ding, X. Yuan, and S. C. Liew, "Network-coded fronthaul transmission for cache-aided C-RAN," in ISIT, June 2017.

[10] J. S. P. Roig, F. Tosato, and D. Gündüz, "Storage-latency trade-off in cache-aided fog radio access networks," 2018. [Online]. Available: http://arxiv.org/abs/1802.01983

[11] A. M. Girgis, O. Ercetin, M. Nafie, and T. ElBatt, "Decentralized coded caching in wireless networks: Trade-off between storage and latency," in ISIT, June 2017.

[12] J. Zhang and P. Elia, "Fundamental limits of cache-aided wireless BC: Interplay of coded-caching and CSIT feedback," IEEE Trans. on Infor. Theory, vol. 63, no. 5, pp. 3142-3160, May 2017. 


$$
\begin{aligned}
g & \leq \frac{\frac{N_{T}-1}{N_{T}}\left(1-\mu_{R}\right)+\frac{1-\mu_{R}}{N_{T} \mu_{R}}\left[1-\left(1-\mu_{R}\right)^{N_{R}}\right]+\frac{\left(1-\mu_{T}\right)^{N_{T}}}{r} \frac{1-\mu_{R}}{\mu_{R}}\left[1-\left(1-\mu_{R}\right)^{N_{R}}\right]}{\max _{l_{1} \in\left[N_{R}\right]} \frac{l_{1}\left(1-\mu_{T}\right)^{N_{T}}\left(1-\mu_{R}\right)^{l_{1}}}{r}+\max _{l_{2} \in\left[N_{R}\right]} \frac{l_{2}\left(1-\mu_{R}\right)^{l_{2}}}{\min \left\{l_{2}, N_{T}\right\}}} \\
& <\frac{\left.12 \max _{l_{2} \in\left[N_{R}\right]}\right] \frac{l_{2}\left(1-\mu_{R}\right)^{l_{2}}}{\min \left\{l_{2}, N_{T}\right\}}+12 \max _{l_{1} \in\left[N_{R}\right]} \frac{l_{1}\left(1-\mu_{T}\right)^{N_{T}}\left(1-\mu_{R}\right)^{l_{1}}}{r}}{\max _{l_{1} \in\left[N_{R}\right]} \frac{l_{1}\left(1-\mu_{T}\right)^{N_{T}}\left(1-\mu_{R}\right)^{l_{1}}}{r}+\max _{l_{2} \in\left[N_{R}\right]} \frac{l_{2}\left(1-\mu_{R}\right)^{l_{2}}}{\min \left\{l_{2}, N_{T}\right\}}}=12
\end{aligned}
$$

